\title{
Wick Rotation in the Light-Front
}

\author{
J. P. B. C. de Melo ${ }^{a * t}$ E. Gambin ${ }^{b}$ and T. Frederico ${ }^{b}$ \\ ${ }^{a}$ Laboratório de Física Teórica e Computação Científica, Universidade Cruzeiro do Sul, \\ 08060-700 and Instituto de Física Teórica, 01405-900, São Paulo, Brazil \\ ${ }^{b}$ Dep. de Física, Instituto Tecnológico de Aeronáutica, 12.228-900, São José dos Campos, Brazil \\ E-mail: pacheco@ift.unesp.br
}

\begin{abstract}
We study the electroweak properties of pseudo-scalar mesons in the light and heavy-light sectors. In particular, we address the electromagnetic form factors and decay constants of the pion, kaon and D mesons. The structure of composite systems are given by the Bethe-Salpeter (BS) amplitude of a meson formed by a confined pair of constituent quark and antiquark, which in our work is written in terms of Pauli-Villars regulators. The analytical structure contains poles in the complex momentum plane. The BS amplitude takes into account poles due to the regulator parameters, while the quark-antiquark cut is avoided, implying in confined quarks with the property that the sum of the constituents masses is larger than the mass of the meson. The one-loop expressions of the electroweak transition amplitudes are conveniently written in terms of light-front momentum. Technically, we introduce a Wick-rotation of the minus component of the momentum $\left(k^{-}\right)$in the one-loop amplitude allowing to avoid the cuts in the complex plane of this momentum variable without crossing them. This is particularly useful as we can study the electroweak properties with several models of the BS amplitude with different powers of Pauli-Villars regulators. The possibility to change the power of the BS amplitude is interesting in order to test the asymptotic behavior of the electromagnetic form factors searching for a suitable form that incorporates the expected QCD decaying power-law form. The results are compared with other models in the literature and with the experimental data.
\end{abstract}

LIGHT CONE 2008 Relativistic Nuclear and Particle Physics

July 7-11 2008

Mulhouse, France

\footnotetext{
* Speaker.

${ }^{\dagger}$ Iftcc.unicsul.com.br
} 


\section{Introduction}

Quantum Chromodynamics is successful in describing subatomic processes for medium and large energies when short distances are probed, and asymptotic freedom justifies the use of perturbative amplitudes. The interesting physics that is investigated in the electroweak structure of hadrons are in part of nonperturbative origin. The understanding of the nonperturbative structure of hadrons with QCD demands a lot of effort and insights from phenomenological models that parameterizes the main properties of confined systems fitted to data. In this respect, relativistic constituent models formulated in the different forms of dynamics proposed by Dirac [1] can be used to describe electroweak transitions. In particular the light-front (LF) form of dynamics, is well known by its maximal number of kinematical boost generators and the stability of the Fock-space truncation under such boosts. Even this limited covariance property is essential for the calculation electroweak observables and in the definition of the partonic content of the hadrons [2]. Therefore, the description of a covariant transition amplitude that relies on nonperturbative hadron BS amplitudes can be decomposed in terms of matrix elements of operators in the light-front Fock-space. In principle it is possible to rewrite a matrix element in terms of the matrix element of an effective operator acting in the valence sector of the hadron. Then, a model that is able to give a LF valence wave function adequate for describing electroweak observables can be useful phenomenological tool to interpret results from nonperturbative calculations of QCD.

In this work, we study the electroweak properties of pseudo-scalar mesons in the light and heavy-light sectors. In particular, we address the electromagnetic form factors and decay constants of the pion, kaon and D mesons. The composite systems are modeled by Bethe-Salpeter amplitudes written in terms of Pauli-Villars regulators, i.e., the analytical structure contains single poles in the complex momentum space. The mesonic Bethe-Salpeter (BS) model amplitude takes into account poles due to the regulator parameters, while the quark-antiquark cut is avoided, implying in confined quarks with the property that the sum of the constituents masses can be smaller than the mass of the meson.

We adopt the light-front momentum as a tool to calculate four-dimensional transition amplitudes involving mesons described as confined systems of a constituent quark and antiquark. Technically, we introduce a Wick-rotation of the minus component of the momentum $\left(k^{-}=k^{0}-k^{3}\right)$ in the one-loop amplitudes allowing to avoid the cuts in the complex plane of this momentum variable without crossing them. This is particularly useful once we can study the electroweak properties of pseudoscalar mesons with several models of the Bethe-Salpeter amplitude with different powers of Pauli-Villars regulators. The possibility to change the power of the BS amplitude is interesting for testing the asymptotic behavior of the electromagnetic form factors and search for a suitable form that incorporates the expected QCD decaying power-law form. The results are compared with other models in the literature and with the experimental data.

\section{The Model: Bethe-Salpeter Amplitude}

The model of the vertex meson $-q \bar{q}$ utilized to construct the Bethe-Salpeter amplitude is

$$
\Lambda_{M}(k, p)=\frac{\left(k^{2}-m_{1}^{2}\right) \Gamma_{M}\left((p-k)^{2}-m_{2}^{2}\right)}{\left(k^{2}-\lambda^{2}+\imath \varepsilon\right)^{n}\left((p-k)^{2}-\lambda_{M}^{2}+\imath \varepsilon\right)^{n}},
$$


where, $\lambda_{M}$ is the scale associated with the meson light-front valence wave function and $n$ is the power of the regulator. $m_{1}$ and $m_{2}$ are the quark and anti-quark masses within the meson bound state. The factors $\left(k^{2}-m_{1}^{2}\right)$ and $\left((p-k)^{2}-m_{2}^{2}\right)$ on the numerator of the vertex function, $\Lambda_{M}(k, p)$, avoids the cuts due the $q \bar{q}$ scattering if $m_{1}+m_{2}$ is smaller than the meson mass. In order to confine the quarks, $\lambda_{M}$ obeys the condition $2 \times \lambda_{M}$ is larger than the mass of the meson.

\section{One-loop electroweak transition amplitudes}

The electromagnetic form factor of a pseudoscalar meson is given by the Mandelstam formula:

$$
\left\langle p^{\prime}\left|J_{q}^{\mu}\left(q^{2}\right)\right| p\right\rangle=\frac{N_{c}}{(2 \pi)^{4}} \int d^{4} k \operatorname{Tr}\left[\Lambda_{M^{\prime}}\left(k, p^{\prime}\right) S_{F}\left(k-p^{\prime}\right) J_{q}^{\mu} S(k-p) \Lambda_{M}(k, p) S_{F}(k)\right],
$$

where $S_{F}(p)$ is the Feynman propagator of the quark with the constituent mass $m_{q}$ and $\Lambda_{M}$ is the meson $-q \bar{q}$ vertex function presented in the last section. $N_{c}=3$ is the number of quark colors. $p^{\mu}$ and $p^{\prime \mu}$ are the initial and final momenta of the system; $q^{\mu}$ is the momentum transfer.

The pseudoscalar electromagnetic form factor is calculated from the matrix element of the electromagnetic current:

$$
\left\langle p^{\prime}\left|J_{q}^{\mu}\left(q^{2}\right)\right| p\right\rangle=\left(p+p^{\prime}\right)^{\mu} F_{P S}^{e m}\left(q^{2}\right) .
$$

The weak decay constant of the pseudoscalar mesons is written as

$$
\left\langle 0\left|A^{\mu}(0)\right| p\right\rangle=\imath \sqrt{2} f_{p s} p^{\mu},
$$

where $A^{\mu}=\bar{q}(x) \gamma^{\mu} \gamma^{5} \frac{\tau}{2} q(x)$. The final expression for the pseudoscalar decay constant is:

$$
\iota p^{\mu} f_{\pi}=\frac{m}{f_{\pi}} N_{c} \int \frac{d k^{4}}{(2 \pi)^{4}} \operatorname{Tr}\left[\gamma^{\mu} \gamma^{5} S(k) \gamma^{5} S(k-p)\right] \Lambda_{M}(k, p) .
$$

We use the plus component of the electromagnetic current, $\gamma^{+}=\gamma^{0}+\gamma^{3}$, to calculate the form factor of the pseudoscalar mesons. In the next section, the Wick rotation for the light-front approach is explained.

\section{Light-Front Wick Rotation}

The Wick rotation in the instant form quantum field theory, is realized by the change in the component $k_{0}$ of the quadri-momentum to $k_{0} \longrightarrow \imath k_{0}$, it is equivalent to a change to the Euclidian space [3]. The original idea was proposed by Wick [4], where the relativistic quantum field theory built with Minkowski spacetime is replaced by the Euclidian space, with the following transformation $\tau=\imath t$, then, the spacetime metric is written as $d s^{2}=d \tau^{2}+d x^{2}+d y^{2}+d z^{2}$. The Wick rotation was applied to solve the Bethe-Salpeter bound state equation (BS) in the Euclidian space, because, the original BS equation formulated in Minkowski space has singularities making it difficult to solve.

In light-front quantum field theory, the choice of frame used to calculate Feynman amplitudes is important, because the position of the poles in the $k^{-}$complex plane from the analytical structure of the integrand are evidently frame dependent. A common frame used to compute form factors 
in the light-front approach is the Breit-frame with the Drell-Yan condition, $q^{+}=0$. In the past, this frame was believed free of contributions from pair terms or zero modes, but this is not really true. Not only the frame is important but also the component of the electromagnetic current used to extract the observables. This was evident after the works done in the references [8, 9]. For example, the pion form factor was calculated with the plus and minus component of the electromagnetic current [8]. The minus component of the current, besides the valence contribution has a nonvalence one, while the plus component of the current has just the valence one. The calculated matrix elements of the electromagnetic current is exactly the same one obtained in an equal time calculation or instant form approach.

The utility of the Wick rotation within the light-front approach is to avoid the singularities related with the poles of the BS amplitudes in computation of the form factor and decay constant. In particular, for the form factor calculation we adopt $q^{+}=-q^{-}=\sqrt{-q^{2}}$. In this frame the poles of integrand of (3.1) in the $k^{-}$complex plane are schematically depicted in figure 1 . This allows to deform the contour from the real axis to the complex plane as shown in the figure. The integration of $k^{-}$runs from $-\infty$ to $+\infty$ and in this case the arc contribution to $J^{+}$vanishes. Indeed, we performed calculations with $\theta$ between $30^{\circ}$ and $90^{\circ}$ and we found no dependence on the rotation angle. This allowed us to perform the Wick rotation of the $k^{-}$momentum in the computation of the form factor with confidence. An analogous analysis can be done for the integrand of (3.4) for the decay constant, with the choice of the rest frame and using the plus component of the axial current.

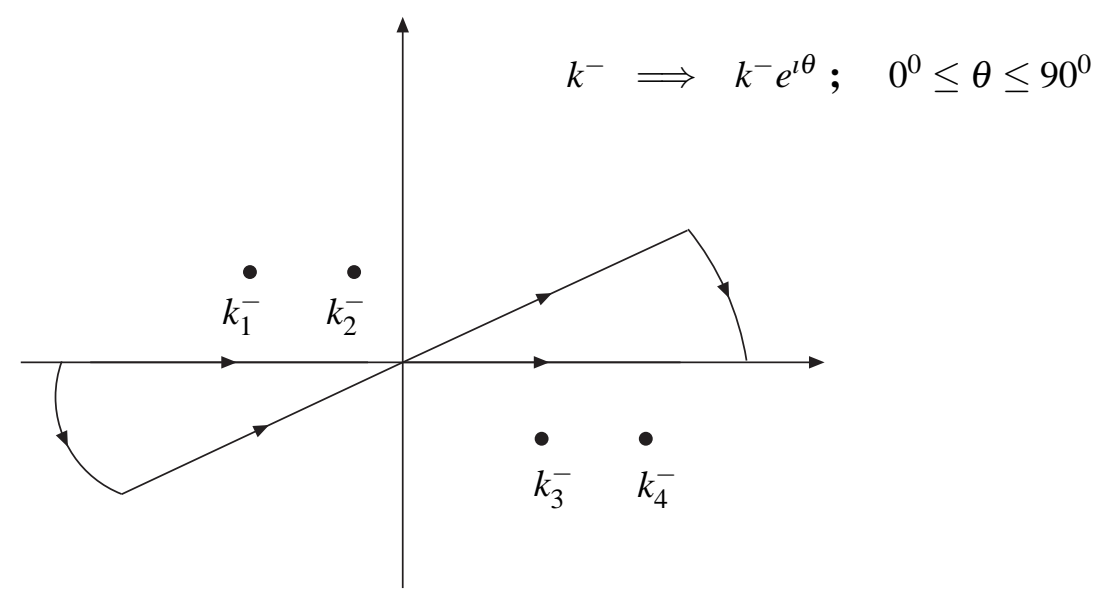

Figure 1: Wick rotation in the minus momentum component.

\section{Results and Conclusion}

We have calculated with the presented confining model the electromagnetic form factors and the weak decay constants for the light pseudoscalar mesons, pion, kaon and $D^{+}$. We used the Wick rotation method performed within the light-front approach, as explained in the last section. The present model will be compared in the following, with other light-front models and dispersion relation calculations $[7,8,10]$. 


\begin{tabular}{|l|l|l|l|l|}
\hline \hline & $\mathrm{n}$ & $r_{P S}(f m)$ & $f_{P S}(\mathrm{MeV})$ & $\lambda_{M}(\mathrm{MeV})$ \\
\hline Pion $(139 \mathrm{MeV})$ & 2 & 0.576 & 92.4 & 542 \\
$m_{u}=220(\mathrm{MeV})$ & 3 & 0.494 & 92.4 & 926 \\
$m_{d}=220(\mathrm{MeV})$ & 4 & 0.456 & 92.4 & 1255 \\
\hline Exp. (Pion) & & 0.672 & 92.42 & \\
\hline Kaon (494 MeV) & 2 & 0.474 & 113 & 648 \\
$m_{s}=0.508(\mathrm{MeV})$ & 3 & 0.453 & 113 & 933 \\
$m_{u}=0.220(\mathrm{MeV})$ & 4 & 0.450 & 113 & 1156 \\
\hline Exp.(Kaon) & & 0.560 & 113 & \\
\hline \hline
\end{tabular}

Table 1: Results for the pseudoscalar weak decay constants $f_{p s}$ and the electromagnetic radius for the pion and kaon compared with the experimental data.

The parameters of the model are: the constituents quark masses, the power $n$ of the vertex function and the mass scale $\lambda_{M}$. The masses of the constituents quarks are $m_{u}=0.220 \mathrm{GeV}$, $m_{s}=0.508 \mathrm{GeV}$, and $m_{c}=1.623 \mathrm{GeV}$. The mass scale parameter is obtained from the fit of the experimental value of the weak decay constant $f_{p s}$.

The table 1 shows the results for the electromagnetic radius and weak decay constants for the pion and kaon, compared with the experimental data. The value of the electromagnetic radius of the pion is approximately $14 \%$ below of the experimental value $(0.675 \pm 0.02)$ [11] for $n=2$, while by increasing $n$ the radius becomes smaller and the mass scale increases. The electromagnetic form factor for the pion is presented in fig. 2; and the results for the kaon and $D^{+}$are shown in fig. 3.

In conclusion, the possibility to change the power of the BS amplitude is interesting in order to test the asymptotic behavior of the electromagnetic form factors searching for a suitable form that incorporates the expected QCD decaying power-law form. We have presented several calculations of the electromagnetic form factors and weak decay constants of the pion, kaon and $D^{+}$with a confining light-front model. Our approach allows to perform easily the numerical calculations with these different light-front models. We have also presented a comparison of our new results with other models and with the experimental data.

We thank FAPESP and CNPq for partial financial support.

\section{References}

[1] P. Dirac, Rev. Mod. Phys. 21, 392 (1949).

[2] Stanley J. Brodsky, Hans-Christian Pauli and Stephen S. Pinsky, Phys.Rept. 301, (1998)299. .

[3] C. Itzykson and J. Zuber, Quantum Field Theory, McGraw-Hill Book Co., (1980).

[4] G. C. Wick, Phys. Rev. 96, 1124 (1954).

[5] J. P. B. C. de Melo and T. Frederico, Phy. Rev. C55, 2043 (1997).

[6] H. W. L. Naus, J. P. B. C. de Melo and T. Frederico, Few-Body Syst. 24, 99 (1998).

[7] J. P. B. C. de Melo, T. Frederico, E. Pace and G. Salmé, Nucl. Phy. A707, 399 (2002).

[8] J. P. B. C. de Melo, H. W. Naus and T. Frederico, Phys. Rev. C59, 2278 (1999). 

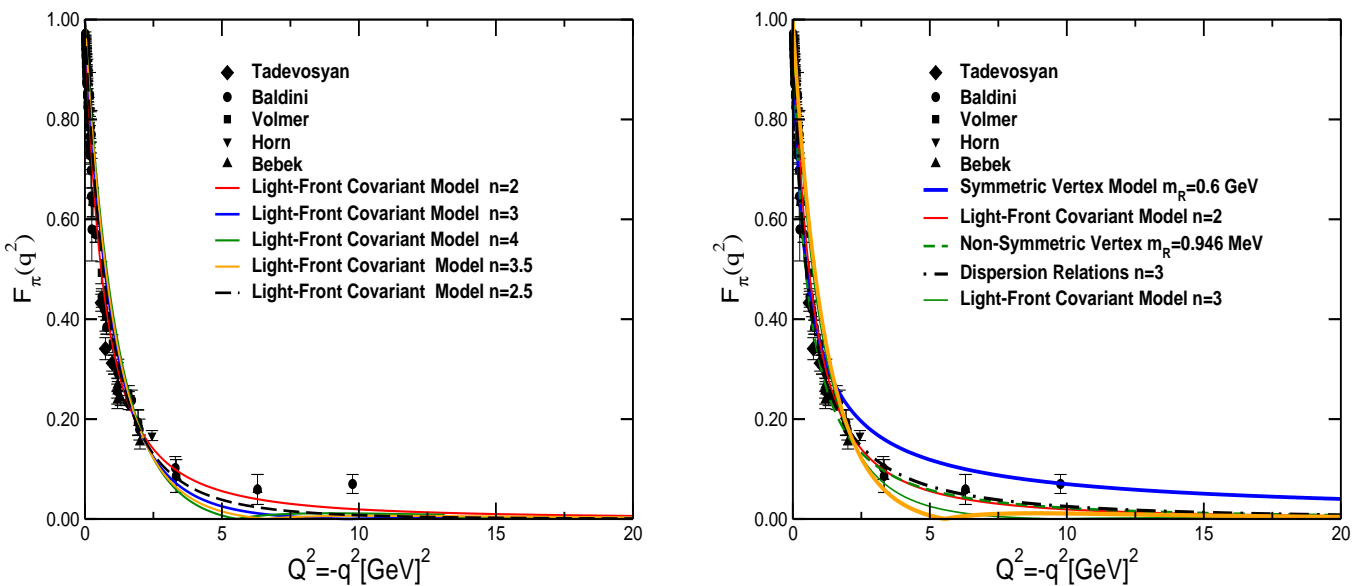

Figure 2: The left plot shows the pion electromagnetic form factor calculated with the light-front covariant model for different values of $n$ and it is compared to the experimental data [12]. In the right plot, the lightfront covariant model is compared with other light-front models: symmetric [7], non-symmetric [8] and dispersion relations [10].
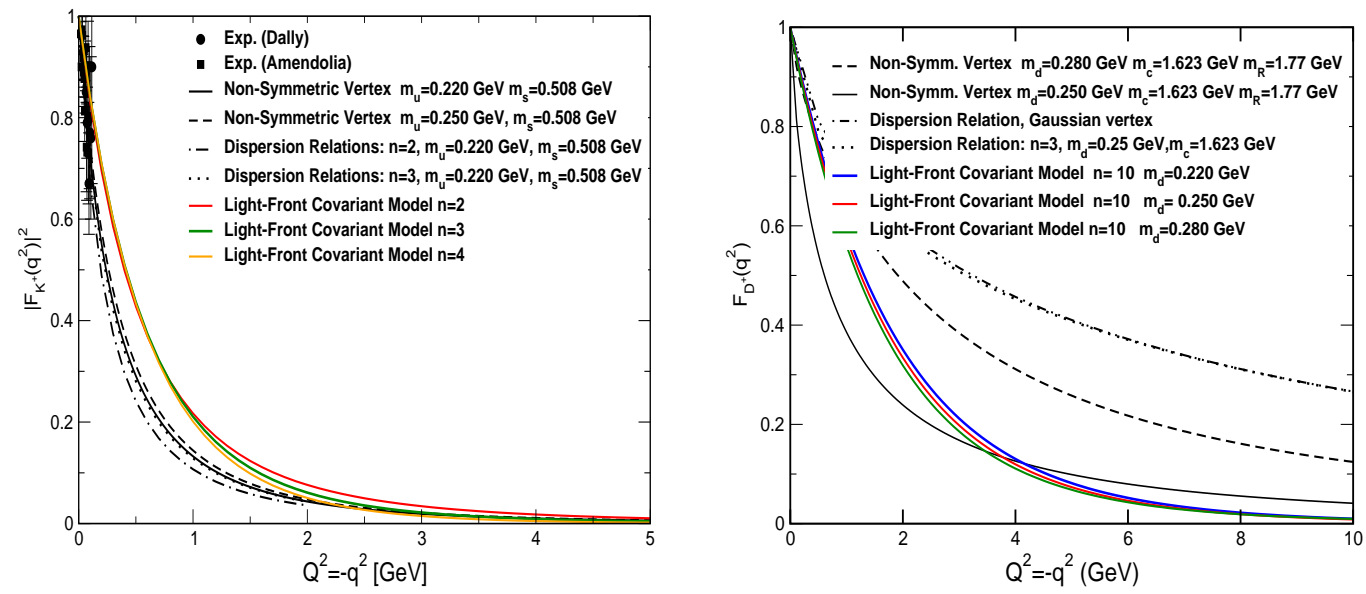

Figure 3: The kaon electromagnetic form factor (left) calculated with light-front covariant model and other models is compared with the experimental data $[11,13]$. The electromagnetic form factor for the $D^{+}$meson with the covariant light-front model and compared with other models (see the label of fig. 2).

[9] B. L. G. Bakker, H. M. Choi, C. -R. Ji, Phys. Rev. D63, 074014 (2001).

[10] B. El-Bennich, J. P. B. C. de Melo, B. Loiseau, J.-P. Dedonder and T. Frederico. Brazilian Journal of Physics, Vol. 38, 465 (2008).

[11] S. R. Amendolia et al., Phys. Lett. 178, 116 (1986).

[12] R. Baldini et al., Eur. Phys. J. C 11709 (1999); Nucl. Phys. A 666\& 667, 3 (2000); J. Volmer et al., Phys. Rev. Lett. 86, 1713 (2001); T. Horn et al., Phys. Rev. Lett. 97, 192001 (2006); V. Tadevosyan et al., Phys. Rev. C 75, 055205 (2007).

[13] S. R. Amendolia et al., Phys. Lett. B277, 168 (1986). 